\title{
The second order concentration corrections to the mutual diffusion coefficient of Brownian macroparticles ${ }^{a)}$
}

\author{
George D. J. Phillies \\ Department of Chemistry, The University of Michigan, Ann Arbor, Michigan 48109 \\ (Received 4 May 1982; accepted 20 May 1982) \\ From the initial time dependence of the dynamic structure factor $S(k, t)$, we obtain a general form, valid \\ through second order in the concentration, for the mutual diffusion coefficient $D_{m}$. All effects of direct and \\ hydrodynamic interactions (other than dynamic friction) are taken into account, including the three-point \\ "Oseen" hydrodynamic tensor $T_{i j}$, whose analytic form is obtained here for the first time. The concept of the \\ reference frame correction is re-examined. The usual factor $(1-\phi)$ for transition from the fundamental \\ hydrodynamic frame to the volume-fixed frame is argued to be a low-concentration approximation. The \\ general form for $D_{m}$ is evaluated for a model system of hard spheres. With the use of the auxiliary assumption \\ (relaxed herein) that all hydrodynamic interaction tensors satisfy $\nabla \cdot t=0$, use of our new general method [J \\ Stat. Phys. 28, 673 (1982)] for reducing $N$-particle cluster integrals to $(N-1)$-dimensional integrals shows \\ $D_{m}=D_{0}\left(1-0.875 \phi-19.53 \phi^{2}\right)$.
}

\section{INTRODUCTION}

Extensive calculations have been made of the spectrum $S(\mathbf{k}, t)$ of light scattered quasielastically by a solution of interacting Brownian macroparticles. ${ }^{1-6}$ With few exceptions, these calculations have only been used to obtain corrections of order $c_{0}^{1}$ to the free diffusion form for $S(\mathbf{k}, t)$. Recently, physical tests of these calculations have been reported. ' These are extremely difficult experiments; at low macroparticle concentration $c_{0}$, the effect of concentration on the normalized initial slope $K_{1}$ of $S(\mathbf{k}, t)$ is small. By carrying out such studies at higher macroparticle concentrations, the concentration dependence of $K_{1}$ ought to be more readily apparent. How ever, significant obstacles lurk at larger $c_{0}$ : (i) multiple scattering may distort the observed spectrum $S_{0}(\mathbf{k}, t)$; (ii) as the magnitude of the second order correction to $K_{1}$ is unknown, it is not clear how large $c_{0}$ may be made without taking $c_{0}^{2}$ (i.e., three-particle) terms into account.

Two rigorous methods of removing the first of these obstacles exist. First, if solvent and solute are suffi ciently similar in index of refraction, ${ }^{7}$ only single scattering will occur. Second, it has recently been demonstrated ${ }^{8}$ that by using a two-beam, two-detector light scattering spectrometer, multiple scattering effects may be removed from the spectrum.

This paper deals with the second obstacle. The conventional effects of direct and hydrodynamic interactions on $K_{1}$, including all terms of second order in the concentration, are here calculated. Formal expressions are given for $K_{1}$ in terms of the hydrodynamic interaction tensors and the two-and three-particle radial distribution functions; these expressions are evaluated for the traditional model suspension of hard spheres.

Section II presents our general method of calculation, which is based on the Langevin equation. Comparison is made with other, ${ }^{9}$ nearly equivalent, methods for making the needed time series expansion. Section III of this paper obtained the form of the three-point hydro-

\footnotetext{
a) This work supported in part by the National Science Founda-
} tion under Grant CHE 79-20389. dynamic interaction, using methods similar to those of Felderhof. ${ }^{10}$ Section IV of this paper applies these results to determine the initial slope of $S(\mathbf{k}, t)$, including both general expressions in terms of equilibrium dis tribution functions and specific results for the hardsphere suspension. The calculation is discussed in Sec. $\mathrm{V}$. The Appendices present our method of introducing the reference frame correction ${ }^{11}$ and exhibit the mathematical methods used to treat our three-point integrals. These methods, which are based closely on a procedure demonstrated in many-electron quantum theory by Sil verstone and Moats, ${ }^{12}$ allow one to reduce $N$-point cluster integrals to $(N-1)$-dimensional integrals over power series. ${ }^{13}$

\section{A TIME SERIES EXPANSION FOR $S(k, t)$}

In the absence of multiple scattering, the quasi elastic light scattering spectrum is directly sensitive to the intermediate scattering function (dynamic structure factor)

$$
S(\mathbf{k}, t)=\left\langle\frac{1}{N} \sum_{i, j=1}^{N} \exp \left\{i \mathbf{k} \cdot\left[\mathbf{r}_{j}(0)-\mathbf{r}_{i}(t)\right]\right\}\right\rangle,
$$

where $\mathbf{k}$ is the scattering vector, $\mathbf{r}_{i}(t)$ denotes the position of the $i$ th of the $N$ particles in the scattering volume, and the brackets " $\langle$ " denote a time or ensemble aver age. The particle displacements are trivially related to the velocities

$$
\mathbf{r}_{i}(t)-\mathbf{r}_{i}(0)=\int_{0}^{t} d s \mathbf{v}_{i}(s)
$$

Substitution of Eq. (2.2) into Eq. (2.1) and expansion of the exponential into a power series gives

$$
\begin{aligned}
S(\mathbf{k}, t)= & \frac{1}{N}\left\langle\sum_{i, j=1}^{N} \exp \left\{i \mathbf{k} \cdot\left[\mathbf{r}_{j}(0)-\mathbf{r}_{i}(0)\right]\right\}\right. \\
& \left.\times \sum_{n=0}^{\infty} \frac{1}{n !}\left[i \mathbf{k} \cdot \int_{0}^{t} \mathbf{v}_{i}(s) d s\right]^{n}\right\rangle .
\end{aligned}
$$

To proceed further, the physical situation needs to be considered in detail. First, $S(\mathbf{k}, t)$ is generally measured by digital autocorrelation, with a highest time resolution of 50-100 ns. Processes which occur on time 
scales much shorter than $50 \mathrm{~ns}$ are seen experimentally only in some average way. Equivalently, in obtaining the "initial" slope

$$
K_{1}=\lim _{t \rightarrow 0}\left[\frac{\partial \ln S(\mathbf{k}, t)}{\partial t}\right],
$$

the limit $t \rightarrow 0$ will actually not be sensitive to the behavior of $2 S(\mathbf{k}, t) / \partial t$ for $t \lesssim$ tens of nanoseconds.

Second, to evaluate Eqs. (2.3) and (2.4), a physical model for the particle motions is required. This paper uses a generalized Langevin equation

$$
m \frac{d \mathrm{v}_{i}}{d t}=-f_{0} \mathbf{v}_{i}+\mathbf{F}_{B i}-\nabla_{i}\left(W_{N}\right)
$$

Here, $m$ and $f_{0}$ are the mass and drag coefficients of particle $i, W_{N}$ is the total potential energy for interactions between the $N$ Brownian macroparticles, and $\mathbf{F}_{B i}$ is the random force of the solvent on the $i$ th macroparticle. This paper will only be concerned with relatively long times, so that $m \dot{v}_{i}=0$ and

$$
\mathbf{v}_{i}=\mathbf{v}_{B i}-f_{0}^{-1} \nabla_{i} W_{N} \text {. }
$$

$\mathbf{v}_{B i}$ is the Brownian velocity of the particle: the component of the particle velocity not accounted for by the interactive velocity $-f_{0}^{-1} \nabla_{i} W^{N}=v_{I i}$. In the simple Langevin model,

$$
\left\langle\mathrm{v}_{B i}(0) \mathrm{v}_{B i}(t)\right\rangle=2 \mathrm{D}_{0} \delta(t) \text {. }
$$

[Real velocity autocorrelation functions do not decay in zero time, so the delta function of Eq. (2.7) is actually a very short-lived time kernel. Correspondingly, the fluctuation-dissipation equation indicates that $f_{0}$ has a high frequency response, rather than being a simple constant; e.g., Eq. (2.5) should properly contain a memory integral $\int d \tau f(t-\tau) v_{i}(\tau)$. It is here assumed that all of these high-frequency effects, to the extent they are significant, are in the kernel which properly replaces $\delta(t)$. While this approximation is imperfect, the use of Eqs. (2.6) and (2.7) (or the related $N$-particle Smoluchowski equation) is almost universal.]

Taking these two considerations into account, substitution of Eq. (2.3) into Eq. (2.4) gives

$$
\begin{aligned}
K_{1}= & +\lim _{t \rightarrow 0^{\prime \prime}}[S(\mathbf{k}, 0)]^{-1} \frac{1}{N}\left\langle\sum_{i, j=1}^{N} \exp \left[i \mathbf{k} \cdot \mathbf{r}_{j i}(0)\right]\right. \\
& \left.\times\left[-i \mathbf{k} \cdot \mathbf{v}_{i}(t)-\int_{0}^{t} \mathbf{k} \cdot \mathbf{v}_{i}(s) \mathbf{k} \cdot \mathbf{v}_{i}(t) d s \cdots\right]\right\rangle .
\end{aligned}
$$

The particle velocities $\mathbf{v}_{i}(t)$ are actually well-behaved continuous functions of time. If the true limit $t \rightarrow 0$ were to be taken, by the usual rules of the calculus the displayed integral in Eq. (2.8) would vanish. However, the limit " $t \rightarrow 0$ " only takes one to times far longer than the very small time $\tau_{B}$ over which $\delta(t)$ of Eq. (2.7) is nonzero. At times $t \gg \tau_{B}$, the integral in Eq. (2.8) includes the entire range of the delta function of Eq. (2.7). The integral over $d s$ and the delta function effectively cancel each other, so in the limit $t \rightarrow 0$ the nonvanishing terms of Eq. (2.8) are

$$
\begin{aligned}
K_{1}= & +\lim _{{ }^{t} t \rightarrow 0^{\prime \prime}}[S(\mathbf{k}, 0)]^{-1} \frac{1}{N}\left\langle\sum _ { i , j = 1 } ^ { N } \operatorname { e x p } [ i \mathbf { k } \cdot \mathbf { r } _ { j i } ( 0 ) ] \left[-i \mathbf{k} \cdot \mathrm{v}_{I i}(t)\right.\right. \\
& \left.-i \mathrm{k} \cdot \mathrm{v}_{B i}(t)\right]-\mathrm{k}^{2}: \int_{0}^{t} d s\left[\mathbf{v}_{B i}(s) \mathbf{v}_{B i}(t)+\mathrm{v}_{B i}(s) \mathrm{v}_{I i}(t)\right. \\
& \left.\left.+\mathrm{v}_{I i}(s) \mathrm{v}_{B i}(t)+\mathrm{v}_{I i}(s) \mathrm{v}_{I i}(t)\right]\right\rangle .
\end{aligned}
$$

The actual evaluation of Eq. (2.9) appears in Sec. IV.

Langevin-type approaches have been used to derive a variety of equation $s^{9}$ similar but not equivalent to Eq. (2.9). In most of these treatments, the integral in Eq. (2.8) is implicitly assumed to vanish, integrals similar to those of Eq. (2.9) being obtained by various indirect means, such as integration of $d^{2} S / d t^{2}$. Since the measurable time derivative of $S(\mathbf{k}, t)$ is

$$
\frac{\delta S(\mathbf{k}, t)}{\delta t}=\tau^{-1}[S(\mathbf{k}, t+\tau)-S(\mathbf{k}, t)]
$$

where $\tau$ is much greater than relaxation time $\tau_{B}$ of the Brownian velocity; it is here presumed that Eq. (2.8) is correct, and that use of the form

$$
\lim _{t \rightarrow 0} \frac{d S(\mathbf{k}, t)}{d t}=\frac{1}{N}\left\langle\sum_{i, j=1}^{N} \exp \left(i \mathbf{k} \cdot \mathbf{r}_{j i}\right)\left(-i \mathbf{k} \cdot \mathbf{v}_{i}\right)\right\rangle
$$

in place of Eq. (2.8) may lead to unreliable results in some circumstances.

\section{THE THREE-POINT HYDRODYNAMIC INTERACTION}

The exact for $m$ for the hydrodynamic interaction between slowly moving spheres in a fluid has been extensively studied by Felderhof ${ }^{10}$ and Batchelor, ${ }^{14}$ who have obtained extensive power series for the diffusion tensors in terms of the center-to-center distance $r$ and the sphere radius $a$. For a pair of spheres, the self - and cross-diffusion tensors are found to be

$$
\begin{aligned}
& \mathbf{D}_{i i}=D_{0}\left[1-\frac{15}{4}\left(\frac{a}{r}\right)^{4} \hat{\mathbf{r}} \hat{\mathbf{r}}+O\left(\frac{a}{r}\right)^{6}\right], \\
& \mathbf{T}_{i j} / f_{0}=\left[\frac{3}{4} \frac{a}{r}(\mathbf{I}+\hat{\mathbf{r}} \hat{\mathbf{r}})+\frac{1}{2}\left(\frac{a}{r}\right)^{3}(\mathbf{I}-3 \hat{\mathbf{r}} \hat{\mathbf{r}})+O\left(\frac{a}{r}\right)^{7}\right] / 6 \pi \eta a,
\end{aligned}
$$

where $D_{0}=k_{B} T / 6 \pi \eta a$ is the free-particle diffusion constant and $\mathbf{I}$ is the identity tensor, the carat $\hat{\mathbf{r}}$ denoting the unit vector along the line of centers of the particles. Surprisingly, in Eq. (3.1b) the $O\left[(a / r)^{5}\right]$ term vanishes.

As this paper is concerned with the $c_{0}^{2}$ corrections to the diffusion coefficient, it is necessary to deal with the effects of hydrodynamic interactions between triplets as well as pairs of spheres. Three-point hydrodynamic interactions do not appear to have been treated previously, but the methods of Felderhof permit their straightforward calculation, at least for low order in $a / r$. Here, we first sketch the two-point treatment and then modify it to examine three-point interactions. Following Fel derhof, the starting point is the Stokes form

$$
\mathbf{v}_{\mathrm{A} 0}(\mathbf{r})=\left[\frac{3}{4} \frac{a}{r}(\mathbf{I}+\hat{\mathbf{r}} \hat{\mathbf{r}})+\frac{1}{4}\left(\frac{a}{r}\right)^{3}(\mathrm{I}-3 \hat{\mathbf{r}} \hat{\mathbf{r}})\right] \cdot \mathbf{F}_{\mathrm{A}} / 6 \pi n a
$$


for the flow $v_{A 0}(r)$ set up at $\mathbf{r}$ by a force $F_{A}$ acting on a sphere A located at the origin. Note that Eqs. (3.2) and (3.1b) are not the same. The flow causes other neighboring spheres to move; if a neighboring sphere $B$ located at $\mathbf{r}_{\mathrm{B}}$ is subject to no other forces, it moves with velocity

$$
\mathrm{U}_{\mathrm{B}}=\mathrm{v}_{\mathrm{A} 0}\left(\mathbf{r}_{\mathrm{B}}\right)+\frac{1}{6} a^{2} \nabla^{2} \mathbf{v}_{\mathrm{A} 0} \text {. }
$$

Substitution of Eq. (3.2) into Eq. (3.3) yields Eq. (3.1b) All further terms arise because spheres $\mathrm{B}$ and $\mathrm{A}$ find themselves in the nonuniform flow field set up by each other. A freely moving sphere $B$, in a nonuniform flow pattern, acts to perturb the flow at points distant from $B$, including the point $r_{A}$ at which sphere $A$ is located. This perturbation $\delta \nabla_{B}(r)$ creates a force on $A$; as linear hydrodynamics are assumed, the change in $\mathbf{v}_{A}$ is given by a formula analogous to Eq. (3.3) but with the indices $A$ and $B$ interchanged.

To obtain the perturbation $\delta \mathbf{v}_{\mathrm{B1}}(r)$ due to $\mathrm{B}$ in the fluid velocity $\mathbf{v}_{A 0}(r)$ created by $A, v_{A 0}(r)$ is subject to a Taylor series expansion at $r_{B}$ :

$$
\begin{aligned}
\boldsymbol{v}_{\mathrm{A} 0}(r)= & \mathbf{v}_{\mathrm{A} 0}\left(\mathbf{r}_{\mathrm{B}}\right)+\left(\mathbf{r}-\mathbf{r}_{\mathrm{B}}\right) \cdot \nabla \mathrm{v}_{\mathrm{A} 0}\left(r_{\mathrm{B}}\right) \\
& +\frac{1}{2}\left(\mathbf{r}-\mathbf{r}_{\mathrm{B}}\right)^{2}: \nabla^{2} \mathbf{v}_{\mathrm{A} 0}\left(\mathbf{r}_{\mathrm{B}}\right)+\cdots .
\end{aligned}
$$

As shown by Felderhof, $\delta \mathbf{v}_{B 1}$ is the sum of the perturbations arising from the field gradient $\left(\mathbf{r}-\mathbf{r}_{\mathrm{B}}\right) \cdot \nabla_{a 0}\left(\mathbf{r}_{\mathrm{B}}\right)$ and each of the higher derivatives of $v_{a 0}(r)$. However, the flow pattern around a sphere in a linear velocity gradient is unique, so if one has for the total flow

$$
\nabla_{B 11}(r)=\left(r-r_{B}\right) \cdot \nabla v_{A 0}\left(r_{B}\right)+\Delta v_{B}(r)
$$

for a system with a sphere at $\mathbf{r}_{\mathrm{B}}$ and a linear flow gradient (where $\Delta v_{B}(r)-0$ as $\left.r-\infty\right), \Delta v_{B}(r)$ is equal to the portion of $\delta \mathbf{v}_{\mathrm{B1}}(\boldsymbol{r})$ arising from the linear gradient term of Eq. (3.4). Equation (3.5) has ${ }^{10}$ the solution

$\mathbf{v}_{\mathrm{B} 11}(\mathbf{r})=\phi(\mathbf{r}) \mathbf{r} \cdot \nabla \mathbf{v}_{\mathbf{A} 0}\left(r_{B}\right)-\frac{1}{3} r^{-1} \phi^{\prime}(\mathbf{r}) \mathbf{r} x\left[\mathbf{r} x(\mathbf{r} \cdot \nabla) \mathbf{v}_{\mathbf{A} 0}\left(\mathbf{r}_{\mathbf{B}}\right)\right]$,

$\phi(r)=1-\frac{5}{2}\left(\frac{a}{r}\right)^{3}+\frac{3}{2}\left(\frac{a}{r}\right)^{5}$,

where to simplify notation the origin in Eq. (3.6) is $\mathbf{r}_{\mathrm{B}}=0$. By combining Eqs. (3.2)-(3.6), one obtains the change in the velocity of $A$, when a force is applied to $A$, due to the presence of $B$, namely,

$$
\delta \mathrm{U}_{\mathrm{A}}=-\frac{15}{4}\left(\frac{a}{r}\right)^{4} \hat{\mathbf{r}} \hat{\mathbf{r}} \cdot \mathrm{F}_{\mathrm{A}} / 6 \pi \eta a+O(a / r)^{6},
$$

as also seen in Eq. (3.1a). Further manipulations give the flow perturbations resulting from the application of bilinear or higher gradients to $\mathrm{B}$; these perturbations are of higher order than $(a / r)^{4}$.

The above reasoning, originally due to Felderhof, is now used for the three-body problem. In the lowest order three-point interaction, a force applied to A establishes a flow at $B$. The disturbance of the flow by $B$ then alters the motion $U_{c}$ of a third sphere $C$. The low est-order modification $\delta v_{B 11}\left(\mathbf{r}_{c}\right)$ to the fluid motion at $\mathbf{r}_{\mathrm{c}}$ may be obtained from Eq. (3.6) if $\mathbf{v}_{\mathrm{A} 0}\left(\mathbf{r}_{\mathrm{B}}\right)$ is interpreted as before, while $\mathbf{r}$ is reinterpreted to mean $\mathbf{r}=\mathbf{r}_{\mathrm{C}}-\mathbf{r}_{\mathrm{B}}$. The change in $\mathrm{U}_{\mathrm{C}}$ caused by $\delta \mathbf{v}_{\mathrm{B} 11}\left(\mathbf{r}_{\mathrm{C}}\right)$ is found from Eq. (3.3), though to lowest order in $(a / r)$ the $\nabla^{2}$ term is not significant. The final result is

$$
\mathbf{T}_{\mathrm{CBA}}=-\frac{15}{8} \frac{a^{4}}{r_{\mathrm{AB}}^{2} r_{\mathrm{BC}}^{2}}\left[1-3\left(\hat{\mathrm{r}}_{\mathrm{AB}} \cdot \hat{\mathbf{r}}_{\mathrm{BC}}\right)^{2}\right] \hat{\mathbf{r}}_{\mathrm{BC}} \hat{\mathbf{r}}_{\mathrm{AB}}
$$

If $\mathrm{C}$ and $\mathrm{A}$ are the same particle, Eq. (3.8) reduces to the aforementioned two-particle form. On inspection, all other three-point interactions, such as the $\mathrm{A} \rightarrow \mathrm{B} \rightarrow \mathrm{C} \rightarrow \mathrm{A}$ or $\mathrm{A} \rightarrow \mathrm{B} \rightarrow \mathrm{C} \rightarrow \mathrm{B}$ couplings, will be of high er order in $a / r_{i j}$, because they require additional gradient operators at $\mathrm{C}$ and further factors of $\phi(r)$.

\section{THE INITIAL SLOPE OF $S(k, t)$}

This section applies the results in Sec. III to evaluate Eq. (2.9). As in our previous paper, ${ }^{6}$ $\left\langle\exp \left[i \mathbf{k} \cdot \mathbf{r}_{j}(0)\right] i \mathbf{k} \cdot \mathbf{v}_{B i}(0)\right\rangle$ is presumed to vanish. The terms of Eq. (2.9) in $\int d t \mathrm{v}_{B i} \mathrm{v}_{H i}$ correspond to the $d y-$ namic friction contribution to the mutual diffusion coefficient. The existence of a dynamic frictional modification to $D_{m}$, at low wave vector in the $t \rightarrow 0$ limit, is in dispute. These terms are treated elsewhere. ${ }^{15-18}$ In the $t-0$ limit $\left\langle\int d s v_{I i}(s) v_{I i}(t)\right\rangle$ vanishes because $v_{I i}$ is smooth and well behaved at small $t$. Using the substitutions

$$
\begin{aligned}
& \mathbf{v}_{I i}=\left(k_{B} T\right)^{-1} \mathbf{D}_{i i} \cdot \mathbf{F}_{i}+\sum_{l \neq i} \mathbf{T}_{i l} \cdot \mathbf{F}_{l} / f_{0}+\sum_{l m} \mathbf{T}_{i m l} \cdot \mathbf{F}_{l} / f_{0}, \\
& \left\langle\mathbf{v}_{B i}(s) \mathbf{v}_{B i}(t)\right\rangle=6 \mathbf{D}_{i i} \delta(s-t),
\end{aligned}
$$

Eq. (2.9) becomes

$$
\begin{aligned}
K_{1}=+\frac{1}{N S(\mathbf{k})} \int \frac{d r^{N} \exp \left[-\beta\left(W_{N}-A\right)\right]}{V^{N}}\left\{\sum_{i, j=1}^{N} e^{i \mathbf{k} \cdot \mathbf{r}_{j i}\left[-i \mathbf{k} \cdot \mathbf{F}_{i} / f_{0}\right]}+\left[\sum_{l=1}^{N}-i \mathbf{k} \cdot \mathbf{b}_{i l}^{s} \cdot \mathbf{F}_{i} / f_{0}\right]\right. & +\sum_{l=1}^{N}\left[-i \mathbf{k} \cdot \mathbf{T}_{i l} \cdot \mathbf{F}_{l} / f_{0}\right] \\
& \left.+\sum_{l, m=1}^{N}\left[-i \mathbf{k} \cdot \mathbf{T}_{i m l} \cdot \mathbf{F}_{l} / f_{0}\right]-\left[\mathbf{k} \cdot D_{0}\left(\mathbf{l}+\sum_{l} \mathbf{b}_{i l}^{s}\right) \cdot \mathbf{k}\right]\right\} \cdot
\end{aligned}
$$

Here, $W_{N}$ is the $N$-macroparticle potential, $f_{0}=6 \pi \eta a, \mathbf{F}$ is the force on particle $i$, the normalization on the ensemble average is

$$
V^{-N} \int d \mathbf{r}^{N} \exp \left[-\beta\left(W_{N}-A\right)\right]=1,
$$

and

$$
\mathbf{b}_{i l}^{s}=-\frac{15}{4}\left(\frac{a}{r_{i l}}\right)^{4} \hat{\mathbf{r}}_{i l} \hat{\mathbf{r}}_{i l}
$$

is the self-component of the hydrodynamic interaction tensor of $i$ in the presence of $l$. In Eq. (4.2), the first 
term in square brackets represents the free drift velocity of each particle $i$ due to the intermacromolecular force on $i$. The second term in square brackets represents the retardation of the drift velocity by the hydrodynamic interaction of $i$ with its neighbors. The third term represents the motion of $i$ due to $i$ 's hydrodynamic interaction (via $\mathbf{T}_{i l}$ ) with some neighboring particle $l$ to which a force has been applied, The fourth term represents the modification of $T_{i l}$, as used in the third term, due to the mediation of some third particle $m$. The final term in square brackets describes the Brownian motion of particle $i$ due to the random stress fluctuations of the solvent as modified by the presence of other particles.

The contributions to $K_{1}$ of these five terms in square brackets will be denoted $\dot{S}_{1}, \dot{S}_{2}, \dot{S}_{3}, S_{4}$, and $\dot{S}_{5}$, respectively. On integration by parts on $\mathbf{F}_{1}=-\nabla_{1} W_{N}$ and $\mathbf{F}_{\boldsymbol{i}}$ $=-\nabla_{i} W_{N}$, resolution of the sums on $i, j, l$, and $m$ into self and distinct parts, and use of the definitions

$$
\begin{aligned}
& V^{-2} g_{2}\left(\mathbf{r}_{i j}\right)=V^{-N} \int d\{N-i j\} \exp \left[-\beta\left(W_{N}-A\right)\right], \\
& V^{-3} g_{3}\left(\mathbf{r}_{i j}, \mathbf{r}_{i t}\right)=V^{-N} \int d\{N-i j l\} \exp \left[-\beta\left(W_{N}-A\right)\right],
\end{aligned}
$$

in which $\mathbf{r}_{i j}=\mathbf{r}_{i}-\mathbf{r}_{j}$ and $d\{N-i j\}$ denotes the integral over all particle coordinates other than $i$ and $j, \mathrm{Eq}$. (4.2) gives

$$
\begin{aligned}
& \dot{S}_{1}+\dot{S}_{2}+\dot{S}_{5}=\frac{-1}{S(\mathbf{k})}\left\{D_{0} k^{2}+c_{0} D_{0} \int d \mathbf{r} g(\mathbf{r})\left[\mathbf{k} \cdot \mathbf{b}^{s}(\mathbf{r}) \cdot \mathbf{k}\right]\right. \\
& \left.\left.\quad-i \mathbf{k} \nabla:\left[\mathbf{b}^{s}(r)\right]\left(e^{-i \mathbf{k} \cdot \mathbf{r}}+1\right)\right]-c_{0}{ }^{2} D_{0} \int d \mathbf{r} d \mathbf{s} i \mathbf{k} \nabla: \mathbf{b}^{s}(r) e^{-i \mathbf{r} \cdot \mathbf{s}} g_{3}(\mathbf{r}, \mathbf{s})\right\}, \\
& \dot{S}_{3}=+[S(k)]^{-1} D_{0} k^{2}\left\{\phi H(k)-c_{0} \int d \mathbf{r} \hat{\mathbf{k}} \cdot \mathbf{T}(\mathbf{r}) \cdot \hat{\mathbf{k}}[g(r)-1] \exp (-i \mathbf{k} \cdot \mathbf{r})\right\} \\
& +[S(k)\}^{-1} V^{-N} D_{0} \int d \mathbf{r}^{N} \exp \left[-\beta\left(W_{N}-A\right)\right] \sum_{\substack{i, l=1 \\
i \neq l}}^{N}\left\{\left[1+\exp \left(i \mathbf{k} \cdot \mathbf{r}_{l i}\right)\right] i \mathbf{k} \nabla_{l}:\left[\mathbf{T}_{i l}\right]+\sum_{\substack{j=1 \\
i, j, i \text { distinct }}}^{N} \exp \left(i \mathbf{k} \cdot \mathbf{r}_{j i}\right) i \mathbf{k} \nabla_{l}:\left[\mathbf{T} \mathbf{T}_{i l}\right]\right\},
\end{aligned}
$$

$$
\begin{aligned}
\dot{S}_{4}= & -[S(\mathbf{k})]^{-1} c_{0}^{2} D_{0} \int d \mathbf{r}_{12} d \mathbf{r}_{32}\left[g_{3}\left(\mathbf{r}_{12}, \mathbf{r}_{32}\right)-1\right] \exp \left(i \mathbf{k} \cdot \mathbf{r}_{31}\right) \mathbf{k} \cdot \mathbf{T}_{123} \cdot \mathbf{k} \\
& +[S(\mathbf{k})]^{-1} V^{-N} D_{0} \int d \mathbf{r}^{N} \exp \left[-\beta\left(W_{N}-A\right)\right] \sum_{\substack{i, l, m=1 \\
i, l, m \text { distinct }}}^{N}\left[1+\exp \left(i \mathbf{k} \cdot \mathbf{r}_{m i}\right)+\exp \left(i \mathbf{k} \cdot \mathbf{r}_{1 i}\right)\right] i \mathbf{k} \nabla_{l}:\left[\mathbf{T}_{i m l}\right]
\end{aligned}
$$

Details of the passage from Eq. (4.2) to Eq. (4.6) are found in Appendix A. In Eq. (4.6a), the integrals in b arise from the terms in

$$
\sum_{i \nabla_{i}}\left[\mathbf{k} \cdot \mathbf{b}_{i l} \exp \left(i \mathbf{k} \cdot \mathbf{r}_{j}\right)_{i}\right]
$$

with $i \neq j=l, i=j \neq l$, and $i \neq j \neq l \neq i$, respectively. In Eq. (4.6b), $\phi$ is the solute volume fraction and $H(\mathrm{k})$ is the spatial Fourier transform of the particle shape. The arguments of Ref. 6 for adding reference frame corrections were used to introduce the $\phi H(\mathbf{k})$ term and to replace $g(\mathbf{r})$ by $g(\mathbf{r})-1$ and $g_{3}\left(\mathbf{r}_{12}, \mathbf{r}_{32}\right)$ by $g_{3}\left(\mathbf{r}_{12}, \mathbf{r}_{32}\right)-1$, as is further treated in Appendix $A$ and the discussion.

The second lines of Eqs. (4.6a) $-(4.6 \mathrm{~b})$ depend on $\nabla \cdot \mathbf{b}, \nabla_{l} \cdot \mathbf{T}_{i l}$, and $\nabla_{l} \cdot \mathbf{T}_{i m l}$, respectively. Many previous workers have assumed that the incompressibility of the fluid implies $\nabla_{l} \cdot \mathbf{b}=\nabla_{l} \cdot \mathbf{T}_{i l}=\nabla_{l} \cdot \mathbf{T}_{i m l}=0$, as may be confirmed for $\mathbf{T}_{i l}$ and $\mathbf{T}_{i \mathrm{ml}}$ by explicit calculation, at least for the terms in $(a / r),(a / r)^{3}$, and $\left(a^{4} / r^{2} r_{1}^{2}\right)$. However, Felderhof ${ }^{10}$ has shown analytically that $\nabla_{l} \cdot \mathbf{T}_{i l}$ does not vanish exactly in its short-range $\left(a^{7} / r^{7}\right)$ terms. This effect may be interpreted as arising from the difference between fluid and rigid body motion: a sphere is limited to rigid translation and rotation, so in a nonuniform flow field the velocities of different points of a freely moving sphere need not equal the unperturbed velocities which the fluid would have, at the same points, if the sphere were absent. Components of $b$ and $T$, which describe the motion of the sphere with respect to the solvent, need not satisfy $\nabla \cdot \mathbf{b}=0=\nabla \cdot T$, which is a requirement for solvent flow. For $T$, this effect is only important in the $(a / r)^{7}$ part of the expansion, which is not being used here. For $\mathbf{b}$, the integrals on $i k \nabla: b$ are nonvanishing even when $b$ is taken from Eq. (3.1a). These terms, which are not small, appear to have been omitted from previous calculations of $K_{1}$, including ours. ${ }^{6}$

The sum of Eqs. $(4.6 a)-(4.6 \mathrm{c})$ gives the mutual diffusion coefficient (the initial slope of $\left.-\dot{S}(\mathbf{k}, t) / k^{2}\right)$ through second order in the concentration, for an arbitrary differentiable interparticle potential $W_{N}$. If $W_{N}$ were not differentiable, the interparticle force $F_{i}$ would be undefined. The applicability of the above approach to a true hard-sphere suspension is therefore not absolutely clear. However, Eqs. (4.6) are correct for a differentiable potential $U_{N}$ which is arbitrarily similar to a hard-sphere potential. The radial distribution functions corresponding to such a $U_{N}$ would be very close to those of the corresponding hard-sphere functions. As long as integrals rather than derivatives of $g(\mathbf{r})$ are required, it does not appear that any fundamental error will be introduced by using hard-sphere distribution functions to obtain approximate solutions to Eqs. (4.6). 
To the extent that differentiability of $W_{N}$ is significant, one notes that real potentials are differentiable, so that results obtained from Eqs. (4.6) are to be preferred to results which depend on the nondifferentiability of the hard-sphere potential.

Equations (4.6) are now solved for the model system. For hard spheres of diameter $\sigma,{ }^{19}$

$$
\begin{aligned}
& g_{2}(r)=0, \quad|\mathbf{r}| \leq \sigma, \\
& g_{2}(r)=1+c_{0} \pi \sigma^{3}\left[\frac{4}{3}-\frac{r}{\sigma}+\frac{1}{12}\left(\frac{r}{\sigma}\right)^{3}\right], \quad \sigma \leq|\mathbf{r}|<2 \sigma,
\end{aligned}
$$

$$
g_{2}(r)=1,|r|>2 \sigma .
$$

To second order in concentration, the Kirkwood superposition approximation for $g_{3}$ is exact, and

$$
\begin{array}{lr}
g_{3}\left(\mathbf{r}_{1}, \mathbf{r}_{2}, \mathbf{r}_{3}\right) \equiv g_{3}\left(\mathbf{r}_{12}, \mathbf{r}_{23}\right)=1 \text { for }\left|\mathbf{r}_{1}-\mathbf{r}_{2}\right| \geq \sigma,\left|\mathbf{r}_{2}-\mathbf{r}_{3}\right| \geq \sigma, \\
\text { and }\left|\mathbf{r}_{1}-\mathbf{r}_{3}\right| \geq \sigma & (4.8 \mathrm{a}) \\
g_{3}\left(\mathbf{r}_{1}, \mathbf{r}_{2}, \mathbf{r}_{3}\right)=0, \text { elsewhere . } & (4.8 \mathrm{~b})
\end{array}
$$

The two-particle integrals in Eqs. (4.6) may be performed by elementary means. The $c_{0}^{2}$ (three-particle) corrections are given by six-dimensional integrals over the interparticle vectors $\mathbf{r}$ and $\mathbf{s}$. As examined in Appendix $\mathrm{B}$, it is possible to reduce an $N$-particle ( $3 N$ dimensional) cluster integral to an $N-1$-dimensional integral while performing only trivial integrations; the nontrivial integrations are found in Appendix $B$. One has for $\mathrm{Eq} .(4.6 \mathrm{a})$ :

$$
\begin{gathered}
\dot{S}_{1}+\dot{S}_{2}+\dot{S}_{5}=\frac{D_{0} k^{2}}{S(k)}\left[(-1)+\left(\frac{15}{8} \phi+1.1083 \phi^{2}\right)\right. \\
\left.+\left(\frac{15}{4} \phi+2.217 \phi^{2}\right)-\left(11.0127 \phi^{2}\right)\right],
\end{gathered}
$$

the parentheses separating contributions from the parts of Eq. (4.6a) which are proportional to $-D_{0} k^{2} / S(k)$, $\int g_{2} \mathbf{k} \cdot \mathbf{b} \cdot \mathbf{k}, \int g_{2} \nabla \cdot \mathbf{b}$, and $\int g_{3} \nabla \cdot \mathbf{b}$, respectively. For Eqs. $(4.6 \mathrm{~b})$ and $(4.6 \mathrm{c})$, one has

$$
\begin{aligned}
& \dot{S}_{3}=\frac{D_{0} k^{2}}{S(k)}\left[\phi H(\mathbf{k})+6 \phi-13.20 \phi^{2}\right], \\
& \dot{S}_{4}=\frac{D_{0} k^{2}}{S(k)}\left[1.63 \phi^{2}\right],
\end{aligned}
$$

or, in total,

$$
\dot{S}=D_{0} k^{2}\left(1+8 \phi+30 \phi^{2}\right)\left[-1+\left(\begin{array}{c}
8.875 \\
12.625
\end{array}\right) \phi-\left(\begin{array}{l}
21.47 \\
19.26
\end{array}\right) \phi^{2}\right],
$$

where, in the coefficients of $\phi$ and $\phi^{2}$, the upper coefficient is correct if $\nabla \cdot \mathbf{b}=0$, while the lower coefficient is valid if one computes $\nabla \cdot b$ directly. From this, one obtains

$$
D_{m}=-\dot{S} / k^{2}=D_{0}\left[1-\left(\begin{array}{l}
0.875 \\
4.625
\end{array}\right) \phi-\left(\begin{array}{c}
19.53 \\
51.74
\end{array}\right) \phi^{2}\right] .
$$

\section{DISCUSSION}

In the above, the second order (three-particle) corrections to the mutual diffusion of a suspension of Brownian macroparticles have been calculated, obtaining the general form given by Eqs. $(4.6 a)-(4.6 c)$. These gen - eral forms were then evaluated for the special case of the hard-sphere suspension, using exact forms for the radial distribution functions of hard spheres. Hydrodynamic interactions between the diffusing particles were included through order $(a / r)^{4}$, using the elegant arguments of Felderhof to calculate the hydrodynamic interaction tensor $\mathbf{T}_{i j l}$. Ignoring terms of higher order in $\mathrm{k}, D_{m}$ was found to be given by Eq. (4.12).

As there appear to be no previous attempts to find the $\phi^{2}$ modification of $D_{m}$, it is not possible to compare the major new results presented here with any in the literature. We therefore reiterate points at which the above work is particularly novel rather than simply tedious. Besides the use of nonadditive terms in the three-point hydrodynamics, the application to nonequilibrium statistical mechanics of Silverstone and Moat's spherical harmonic expansion method ${ }^{12}$ seems likely to have applications other than the one seen here. The threepoint correlation functions often correspond to three-dimensional integrals in which careful work is needed to identify the overlaps of even, e.g., a trio of spheres. As shown in detail in Appendix B, a straightforward procedure has here been demonstrated for simplifying such three-dimensional integrals to two-dimensional integrals with relatively simple (e.g., linear polynomial) bounds.

Our calculation of the $\phi^{1}$ correction to $D_{m}$ is in partial agreement with prior estimates. The values for $\dot{S}_{3}$ and for the "reference frame correction" agree with the literature. Our expression for the $\phi^{1}$ part of $\dot{S}_{1}+\dot{S}_{2}+\dot{S}_{5}$ differs from that previously presented for three reasons. First, hydrodynamic interactions have here been included through $(a / r)^{4}$, while some previous calculations have included corrections through other orders in $(a / r)$. Second, in Eqs. (4.6b) and (4.6c) the hydrody namic interaction tensors $\mathbf{T}$ were systematically aver aged over $g(\mathbf{r})-1$ rather than $g(\mathbf{r})$. In some prior calculations, the $(a / \gamma)^{1}$ part of $\mathbf{T}$ was averaged over $g(\mathbf{r})-1$, but the $(a / r)^{3}$ part of $\mathbf{T}$ was averaged over $g(\mathbf{r})$ itself. These are numerically inequivalent; Appendix A discusses justifications for our choice. Third, other calculations, including one of ours, have either overlooked the $i \mathrm{k} \nabla: \mathbf{b}$ terms or assumed that such terms vanish. The $k \nabla: \mathbf{b}$ terms actually make a substantial contribution to $\dot{S}$. When due allowance is made for these differ ences, the $\phi^{1}$ term of Eq. (4.12) agrees with one in the literature. ${ }^{5,10}$

Historically, ${ }^{11}$ the reference frame correction was set up to correct for the relative motion of the solvent and sample cell, it being assumed that hydrodynamic results for isolated pairs of objects in an infinitely large volume of stationary (at $\mathbf{r} \rightarrow \infty$ ) solvent are essentially the same as the results for a pair of particles in a closed volume in the solvent-fixed frame. The arguments presented in Appendix B raise questions as to the interpretation of this assumption. Except at very short distances, for which $(a / r)^{7} \neq 0$, the velocity of the solute particles is the same as that which the solvent would have had, if the solute had been absent. Within our approximations, in a closed system the solvent and solution-fixed refer ence frames are the same. The displacement of solu tion by a moving particle is seen to modify the diffusion 
rate, but only as a $\phi^{1}$ correction.

While the above calculation is in some respects more detailed than previous studies of this same problem, there are still several in which the treatment in this paper may be incomplete. The role of dynamic friction - the modification of $f_{0}$ by direct interactions in the absence of hydrodynamics - has not been included, though it has previously been shown that dynamic friction has a substantial effect on the drag coefficient. ${ }^{18}$ Furthermore, it is clearly only approximately correct to use continuum hydrodynamics to treat interactions between macromolecules. When $r_{i j}-2 a$ is less than the diameter of a solvent molecule, the continuum form for $\mathbf{T}_{i j}$ must be incorrect. By not including the $(a / r)^{6,7, \ldots}$ corrections to $\mathbf{T}$, this question has in part been avoided. Finally, only the initial slope of $S(k, t)$ has been obtained. The zero-frequency mutual diffusion coefficient deter mines the complete relaxation of a concentration gradient, i.e., it includes the long-term nonexponential behavior of $S(\mathbf{k}, t)$ in nonideal solutions. If $S(\mathbf{k}, t)$ is perturbed by mode-coupling effects, but is dominantly exponential in relaxation, at long times a power law decay of $S(k, t)$ would be expected. The details of this long-time behavior, and the extent to which it would perturb the apparent initial slope $K_{1}$, have not been examined here.

\section{APPENDIX A: REFERENCE FRAME CORRECTIONS AND THE INTEGRATION BY PARTS OF EQ. (4.2)}

In a previous paper [Ref. 6, Eq. (A1)], it was shown that the true hydrodynamic interaction $\mathbf{b}_{i j}^{\prime}$ of two macroparticles $i$ and $j$ satisfies

$$
\int d \mathbf{r}_{i}\left[\mathbf{k} \cdot \mathbf{b}_{i l}^{\prime} \cdot \mathbf{k} \exp \left(i \mathbf{k} \cdot \mathbf{r}_{i j}\right)+\phi_{l} H(\mathbf{k})\right]=0,
$$

$H(k)$ being the spatial Fourier transform of the hydrodynamic shape of either particle, and particle $l$ being taken to drive the flow. Equation (A1) presumes that the particle velocities are equal to the velocities which the fluid would have, at the same locations, if the particles were absent. With the approximations used in Sec. III, this presumption is still satisfied by $\mathbf{T}_{i z}$ and $\boldsymbol{T}_{i j l}$ [Eqs. (3.1b) and (3.8)] as used in this paper, so in Eq. (A1) it is proper to replace

$$
\mathbf{b}_{i j}^{\prime} \Rightarrow \mathbf{T}_{i l}^{\prime}+\sum_{i, j, l \text { distinct }}^{N} \mathbf{T}_{i j l}^{\prime},
$$

$\mathbf{T}_{i l}^{\prime}$ and $\mathbf{T}_{i j l}^{\prime}$ including the modifications of $\mathbf{T}_{i j}$ and $\mathbf{T}_{i j l}$ by the walls of the container. Because the particles simply move with the fluid, the mediating particles of $j$ of $\mathbf{T}_{i j t}^{\prime}$ make no modification to Eq. (A1); only the volume fraction $\phi$ of the particle which initially drives the flow appears in Eq. (A1). Alternatively, unless the particles move significantly with respect to the solvent, $b_{i l}^{\prime}$ may indifferently be said to describe either the response of the solvent to the motions of $l$ or the response of the solution (solvent + solute) to $l$.

We first show how Eq. (A1) is used in the transition from Eq. (4.2) to Eq. (4.6) and then show why $\mathbf{T}_{i j}$ and $\mathbf{T}_{i j l}$ are suitable short-range approximations to $\mathbf{T}_{i j}^{i}$ and $\mathbf{T}_{i j l}^{\prime}$, respectively. Consider the integration by parts which takes one from Eq. (4.2) to Eqs. (4.6b) and (4.6c). By applying to the third and fourth terms of Eq. (4.2) the substitution $\exp \left[-\beta\left(W_{n}-A\right)\right] \mathrm{F}_{1}=-\nabla_{1}\left\{-K_{B} T\right.$

$\left.\times \exp \left[-\beta\left(W_{N}-A\right)\right]\right\}$ and performing the integration, one has

$$
\begin{aligned}
& {[N S(k)]^{-1} V^{-N} \int_{V} d \mathbf{r}^{N} \exp \left[-\beta\left(W_{N}-A\right)\right] \sum_{i, j, l=1}^{N} \exp \left[i \mathbf{k} \cdot \mathbf{r}_{j i}\right]\left[-i \mathbf{k} \cdot \mathbf{T}_{i l}^{\prime} \cdot \mathbf{F}_{l} / f_{0}+\sum_{m=1}^{N}-i \mathbf{k} \cdot \mathbf{T}_{i m l}^{\prime} \cdot \mathbf{F}_{l} / f_{0}\right]} \\
& \left.=[N S(k)]^{-1} D_{0} V^{-N} \int d \mathbf{r}^{N}\left\{\exp \left[-\beta\left(W_{N}-A\right)\right]-C\right]\right\}\left\{\sum_{i, j, l=1}^{N} i \mathbf{k} \nabla_{l}:\left[\exp \left(i \mathbf{k} \cdot \mathbf{r}_{j i}\right) \mathbf{T}_{i l}^{\prime}\right]+\sum_{m=1}^{N} i \mathbf{k} \nabla_{l}:\left[\exp \left(i \mathbf{k} \cdot \mathbf{r}_{j l}\right) \mathbf{T}_{i m l}^{\prime}\right]\right\} \\
& +\int_{s} d \mathbf{S}_{l} \cdot d\{N-l\}\left\{\exp \left[-\beta\left(W_{N}-A\right)\right]-C\right\}\left\{\sum_{i, j, l=1}^{N} \exp \left(i \mathbf{k} \cdot \mathbf{r}_{j l}\right)\left[i \mathbf{k} \cdot \mathbf{T}_{i l}^{\prime}+\sum_{m=1}^{N} i \mathbf{k} \cdot \mathbf{T}_{i m l}^{\prime}\right]\right\}
\end{aligned}
$$

where the final integral represents the surface terms in the integration by parts, $d\{N-l\}$ representing the inte gral over the coordinates of all particles other than $l$. To obtain Eqs. (4.6), the surface terms were presumed to vanish, and the first integral was reorganized.

The choice of an appropriate value for the integrating constant $C$ in Eq. (A3) and its counterparts has sometimes been treated imprecisely. It often has been assumed that the correct choice is $C=1$, which choice as sures convergence of integrals over the long-range part of the Oseen tensor $T_{i j}$. The choice $C=1$ leads to a mathematical difficulty. For the integration by parts to be correct, the integrand must be nonsingular within its range. $T_{i 1}$ and $T_{i m}$ are strongly divergent if $\boldsymbol{r}_{i l}, \mathbf{r}_{i l}$, or $\mathbf{r}_{m i}$ equal zero. This divergence is physically ir relevant, because $T_{i l}$ or $T_{i m}$ then only diverge when particles are within their hard-core radii of each other. While $T \rightarrow \infty$ as $x \rightarrow 0$, one has $g(x)=0$ for $r$ small, so

$$
\lim _{r \rightarrow 0} T_{n}(r) g(r)=0,
$$

and similarly for $T_{i j l}$. By comparison of Eq. (A3) with Eq. (A4), one can see that for the integrand to be well behaved, one must choose $C=0$. In a closed system of arbitrarily large fixed size, the behavior of the true hydrodynamic interaction tensor $T^{\prime}(r)$ at large $r$ insures 
that the surface term of Eq. (A3) vanishes; namely, if the surface of integration $S$ is on or outside of the walls of the container, $\mathbf{T}^{\prime}(\mathbf{S})$ is zero.

The use of $\mathbf{T}^{\prime}$ rather than the Oseen type (i.e., open infinite volume forms) $\mathbf{T}_{i j}$ and $\mathbf{T}_{i j t}$ is clearly inconvenient, as $\mathbf{T}^{\prime}$ depends in an unknown way on the positions of $i$ and $l$ with respect to the walls of the container. While Faxen ${ }^{20}$ has indicated how $\mathbf{T}^{\prime}$ can be obtained, for our purposes a simpler approximation seems appropriate. The difference between $\mathbf{T}^{\prime}$ and $\mathbf{T}$ may be expressed as a power series in $R / L, R$ being the distance from the container wall to the particle and $L$ being a dimension of the container. Over distances $\sim a, \mathbf{T}^{\prime}-\mathbf{T}$ is nearly a constant (except near the walls). Furthermore, $\mathbf{T}^{\prime}-\mathbf{T}$ largely reflects a cancellation of the very longrange part of $T$, which at any point is small (though its effect on $\int \mathbf{d} r \mathbf{k} \cdot \mathbf{T} \cdot \mathbf{k}$ is large), so $\mathbf{T}^{\prime}-\mathbf{T}$ must every where be similar in magnitude to $\mathbf{T}(L)$. If $(r / a)$ is s mall, $\mathbf{T}^{\prime}-\mathbf{T} \approx T(L)$ is $\ll T(r)$, so for $r \ll L$ one may approxiınate $\mathbf{T}^{\prime}$ with the infinite-volume Oseen form $\mathbf{T}$. This approximation is useless in Eq. (A3), which requires $\mathbf{T}$ for large interparticle separations. However, by subtraction of Eq. (A1) [after multiplication by suitable constants and insertion of dummy integrals $\int d \mathbf{r}_{j} V^{-1}$ $(j \neq i, l)], \mathrm{Eq} .(\mathrm{A3})$ is found to be equivalent to Eqs. $(4.6 \mathrm{~b})$ and $(4.6 \mathrm{c})$. Equations $(4.6 \mathrm{~b})$ and $(4.6 \mathrm{c})$ only require $T^{\prime}$ for small values of $r$, so in Eqs. (4.6) the approximation $T^{\prime} \approx T$ is useful. As noted in the dis cussion, the above argument leads to a $\mathbf{T}[g(\mathbf{r})-1]$ term similar to that obtained before in other ways, except from the above one concludes that $[g(r)-1]$ is to multiply the whole of hydrodynamic interaction tensor $\mathbf{T}_{\|}$, not just its long-range $\left[(a / r)^{1}\right]$ part, as is sometimes done. The terms of $T_{i l}$ for which $\int[g-1]$ would prove troublesome at small $r$ are precisely those for which Eq. (A1) is inapplicable. At these higher orders in $(a / r)$, the results of this Appendix are not sufficient.

\section{APPENDIX B: EVALUATION OF THREE-POINT CLUSTER INTEGRALS}

The method used here to compute three-point integrals is based on results of Silverstone and Moats, ${ }^{12}$ who demonstrate that an arbitrary function $F(r)=$ $=f_{L M}(r) Y_{L M}(\theta, \phi)$ can be expanded in terms of spherical harmonics centered on a displaced origin, namely,

$$
\begin{aligned}
F(r-R)= & \sum_{\substack{l=0 \\
l+L+\lambda \text { even }}}^{\infty} \sum_{\lambda=|l-L|}^{l+L} \nu_{l \lambda L}(r, R) \\
& \times \sum_{m=-l}^{l} C_{\lambda L L t l m} Y_{\lambda, \mu-m}\left(\theta_{R}, \phi_{R}\right) Y_{l m}\left(\theta_{r}, \phi_{r}\right) .
\end{aligned}
$$

Here, $R$ is the position of the origin in the initial coor dinate system, and

$$
\begin{aligned}
& C_{\lambda L M l m}=\int d \Omega Y_{\lambda, N-m}^{*}(\Omega) Y_{l m}^{*}(\Omega) Y_{L M}(\Omega), \\
& \nu_{l \lambda L}(r, R)=\frac{2 \pi(-1)^{l}}{R} \sum_{a=0}^{L+l+\lambda / 2} \sum_{b=0}^{(L+l+\lambda-2 a / 2)} D_{l \lambda \text { Lab }}\left(\frac{r}{R}\right)^{2 b-l-1} \\
& \quad \times \int_{\mid r-R !}^{r+R} d r^{\prime}\left(\frac{r^{\prime}}{R}\right)^{2 a-L+1} f_{l m}\left(r^{\prime}\right), \\
& \quad \times(L+l+\lambda-2 a-2 b) ! !(2 b) ! !(L+l-\lambda-2 a-2 b-1) ! !], \\
& D_{l \lambda L \text { Lab }}=1 /[(2 a) ! !(2 a-2 L-1) ! !(2 b-2 l-1) ! ! \\
& (2 N) ! !=2^{N} N !, \\
& (2 N-1) ! !=(2 N) ! /(2 N) ! !, \\
& (-2 N-1) ! !=(-1)^{N} /(2 N-1) ! ! .
\end{aligned}
$$

To use this method to evaluate a multicenter integral, all functions in the integrand are expanded in terms of radial distances from a given center and spherical harmonics fixed on that same center. The angular integrals all involve only products of spherical harmonics, which are trivial, so only the radial integrals require serious analytic effort. Here, we were concerned with hard spheres, for which

$$
\begin{aligned}
& f_{00}(r)=-\sqrt{4 \pi}, \quad|\mathbf{r}|<\sigma ; f_{00}(r)=0, \quad|\mathbf{r}| \geq \sigma, \\
& \nu_{000}(r, s)=\frac{\pi}{r s}\left(\min \left\{\begin{array}{c}
(r+s)^{2} \\
\sigma^{2}
\end{array}\right\}-\min \left\{\begin{array}{c}
(r-s)^{2} \\
\sigma^{2}
\end{array}\right\}\right)(-\sqrt{4 \pi}), \\
& \nu_{u t 0}(r, s)=0 \quad l>0, \quad r+s<\sigma \text { or }|r-s| \leq \sigma, \\
& \nu_{110}(r, s)=\frac{-\pi}{4 r^{2} s^{2}}\left(\sigma^{4}-2 r^{2} \sigma^{2}-2 s^{2} \sigma^{2}-2 r^{2} s^{2}+r^{4}+s^{4}\right)(-\sqrt{4 \pi}), \\
& r+s>\sigma, \quad|r-s| \leq \sigma,
\end{aligned}
$$

and

$$
C_{l 00 l m}=1 / \sqrt{4} \pi(-1)^{m} .
$$

Three-center integrals are needed for Eq. (4.6c) and the final term of Eq. (4.6a). Equation $(4.6 \mathrm{c})$ is the more challenging of these; the same line of attack is effective against $\mathrm{Eq}$. (4.6a). It is most convenient to take particle two as the origin. On introducing the Mayer $f$ function $f_{i j}=g_{2}\left(r_{i j}\right)-1$, the Kirkwood superposition principle gives

$g_{3}\left(\mathbf{r}_{12}, \mathbf{r}_{32}\right)-1=g_{2}\left(\mathbf{r}_{12}\right) g_{2}\left(\mathbf{r}_{32}\right) f_{13}+g_{2}\left(\mathbf{r}_{12}\right) g_{2}\left(\mathbf{r}_{32}\right)-1$.

The complex exponential $\exp \left[i \mathbf{k} \cdot\left(\mathbf{r}_{12}-\mathbf{r}_{32}\right)\right]$ may be expanded in spherical waves by using

$\exp (i \mathrm{k} \cdot \mathbf{r})=4 \pi \sum_{l=0}^{\infty} \sum_{m=-l}^{l} i^{l} j_{l}(k r) Y_{l m}\left(\theta_{k}, \phi_{k}\right) Y_{l m}^{*}\left(\theta_{r}, \phi_{r}\right)$,

$j_{l}(k r)$ being a spherical Bessel function.

By substitution, with $r_{12}=r, r_{32}=s$,

$$
\begin{aligned}
\dot{S}_{4}= & {[S(k)]^{-1} c_{0}^{2} D_{0} k^{2} \int r^{2} d r d \Omega_{r} s^{2} d s d \Omega_{s}\left[g_{2}(r) g_{2}(s) \sum_{a b} \nu_{a a 0}(r, s) C_{a 00 a b} Y_{a,-b}\left(\Omega_{r}\right) Y_{a b}\left(\Omega_{s}\right)+g_{2}(r) g_{2}(s)-1\right] } \\
& \left.\times\left[(4 \pi)^{2} \sum_{c d} \sum_{e f} i^{c-s} j_{c}(k r) j_{e}(k s) Y_{c d}^{*}\left(\Omega_{k}\right) Y_{c d}\left(\Omega_{r}\right) Y_{e f}^{*}\left(\Omega_{k}\right) Y_{e f}\left(\Omega_{s}\right)\right]\right]\left\{\frac{15}{8} \frac{a^{4}}{r^{2} s^{2}}(\hat{\mathbf{k}} \cdot \hat{\mathbf{r}})(\hat{\mathbf{k}} \cdot \hat{\mathbf{s}})\left[1-3(\hat{\mathbf{r}} \cdot \hat{\mathbf{s}})^{2}\right]\right\} .
\end{aligned}
$$


Each sum with two indices is

$$
\sum_{a b}=\sum_{a=0}^{\infty} \sum_{b=-a}^{a}
$$

$\hat{\mathbf{k}}=\mathbf{k} /|\mathbf{k}|$, and in $\mathbf{T}_{\mathbf{C B A}}$ one has $s=-\mathbf{r}_{A B}$. The orientation of the coordinate frame is arbitrary. It is convenient to choose coordinates such that $\theta_{k}=\phi_{k}=0$, since $Y_{l m}(0,0)=0$ for $m \neq 0$. The angular dependence of $\mathbf{T}_{i l m}$, in terms of spherical harmonics, is

$$
\begin{aligned}
(\hat{\mathbf{k}} \cdot \hat{\mathbf{r}})(\hat{\mathbf{k}} \cdot \hat{\mathbf{s}})\left[1-3(\hat{\mathbf{r}} \cdot \hat{\mathbf{s}})^{2}\right]=\pi\left[\frac{4}{3} Y_{10}^{r} Y_{10}^{s}-3\left\{\left(\frac{4}{5 \sqrt{7}} Y_{30}^{r}+\frac{6}{5 \sqrt{3}} Y_{10}^{r}\right)\left(\frac{4}{5 \sqrt{7}} Y_{30}^{s}+\frac{6}{5 \sqrt{3}} Y_{10}^{s}\right)-\frac{1}{25}\left(\frac{8}{\sqrt{21}} Y_{31}^{r}+\sqrt{\frac{8}{3}} Y_{11}^{r}\right)\left(\frac{8}{\sqrt{21}} Y_{3,-1}^{s}+\sqrt{\frac{8}{3}} Y_{1-1}^{s}\right)\right.\right. \\
\left.\left.-\frac{1}{25}\left(\frac{8}{\sqrt{21}} Y_{31}^{s}+\sqrt{\frac{8}{3}} Y_{11}^{s}\right)\left(\frac{8}{\sqrt{21}} Y_{3-1}^{r}+\sqrt{\frac{8}{3}} Y_{1-1}^{r}\right)+\frac{1}{2}\left[\left(\frac{4}{5 \sqrt{3}} Y_{10}^{r}-\frac{4}{5 \sqrt{7}} Y_{30}^{r}\right)\left(\frac{4}{5 \sqrt{3}} Y_{10}^{s}-\frac{4}{5 \sqrt{7}} Y_{30}^{s}\right)\right]+\frac{16}{105}\left(Y_{32}^{r} Y_{32}^{s}+Y_{3-2}^{r} Y_{3-2}^{s}\right)\right\}\right],
\end{aligned}
$$

in which $Y_{32}^{r}=Y_{32}\left(\Omega_{r}\right)$, etc. The angular integrals are given by ${ }^{21}$

$$
\int Y_{l m}(\Omega) Y_{n \phi}(\Omega) Y_{a r}(\Omega) d \Omega=\left[\frac{(2 l+1)(2 n+1)(2 r+1)}{4 \pi}\right]^{1 / 2}\left\{\begin{array}{lll}
l & n & q \\
0 & 0 & 0
\end{array}\right\}\left\{\begin{array}{lll}
l & n & q \\
m & p & r
\end{array}\right\}
$$

The braces " \{\} " denoting Wigner $3-j$ symbols, which vanish for $l+n+q$ odd or $m+p+r \neq 0$. Combining Eqs. (A6) (A9),

$$
\begin{aligned}
& \dot{S}_{4}=\left\{-30 \pi^{2} \frac{D_{0} k^{2}}{S(k)} C_{0}^{2} a^{4}\right\} \int_{\sigma}^{\infty} d r \int_{0}^{\infty} d s \sum_{a b} \sum_{c d} \sum_{e f} \sum_{x y} \sum_{w=\varepsilon}\left[\nu_{a a 0}(r, s) C_{a 00 a b} j_{c}(k r) j_{e}(k s)\left\{Y_{c 0}^{*}(0) Y_{e 0}^{*}(0)\right\}\right. \\
& \left.\times K_{x y z w} \frac{2 a+1}{4 \pi}[(2 c+1)(2 x+1)(2 e+1)(2 z+1)]^{1 / 2}\left\{\begin{array}{lll}
a & c & x \\
0 & 0 & 0
\end{array}\right\}\left\{\begin{array}{lll}
a & c & x \\
b & 0 & y
\end{array}\right\}\left\{\begin{array}{lll}
a & e & z \\
0 & 0 & 0
\end{array}\right\}\left\{\begin{array}{lll}
a & e & z \\
b & 0 & -w
\end{array}\right\}\right],
\end{aligned}
$$

where $K_{x y z w}$ is the coefficient of the $Y_{x y}^{r} Y_{z w}^{s}$ term on the rhs of Eq. (B8). Since $x y, w z$ are limited to the pairs $(1,0),(1 \pm 1),(3,0),(3, \pm 1)$, and $(3, \pm 2)$ the sums on $x y, z w$ include only a minimal number of terms; also, the $3-j$ symbols insure $b=w=-y$.

As this paper is being held to the $\mathrm{k}-0, k \sigma-0$ limit, so that $\dot{S}$ is proportional to $k^{2}$, most terms of Eq. (B10) drop out. Indeed, only the $c=e=0, a=x=z=(1$ or 3$)$ terms are of importance. The $a=1$ term is

$$
\begin{aligned}
S_{4 \mathrm{~A}}= & Q_{1} Q_{2}\left[k^{-2} \int_{\tau}^{2 \tau} d x \int_{\tau}^{\tau+x} d y j_{0}(x) j_{0}(y)\right] \frac{\pi}{4 x^{2} y^{2}}\left[\tau^{4}-2 x^{2} \tau^{2}-2 y^{2} \tau^{2}-2 x^{2} y^{2}+x^{4}+y^{4}\right] \\
& +k^{-2} \int_{2 \tau}^{\infty} d x \int_{x-\tau}^{x+\tau} d y j_{1}(x) j_{1}(y) \frac{\pi}{4 x^{2} y^{2}}\left[\tau^{4}-2 x^{2} \tau^{2}-2 y^{2} \tau^{2}-2 x^{2} y^{2}+x^{4}+y^{4}\right],
\end{aligned}
$$

where $\tau=k \sigma, x=k r$, and $y=k s$, and $Q_{1}$ and $Q_{2}$ denote the coefficients of $S_{4}$ delimited by braces. The integrals on $y$ are in standard references. In the $\tau \rightarrow 0$ limit, the first integral over $x$ may be done by making power series expansions around $x=0$, of $j_{0}(x)$, and of the $\sin (y), \cos (y)$, si $(y)$ terms resulting from $\int d y$, and eliminating monomials of excessive order in $k$. The second integral on $x$ is

$$
\frac{\pi}{8 k^{2}} \int_{2 \tau}^{\infty} d x j_{0}(x)\left[\frac{\sin (x-\tau)}{(x-\tau)^{2}}-\frac{\sin (x+\tau)}{(x+\tau)^{2}}+\frac{\cos (x-\tau)}{(x-\tau)}-\frac{\cos (x+\tau)}{(x+\tau)}+\operatorname{Si}(x-\tau)-\operatorname{si}(x-\tau)\right]
$$

by taking series expansions of the trigonometric functions about $x\left[\right.$ so, e.g., $\left.\sin (x-\tau)=\sin x-\tau \cos x+\frac{1}{2} \tau^{2} \cdots\right]$ it is found that this integral is $0\left(\tau^{4}\right)$, and thus does not contribute to $D_{m}$ in the $k \rightarrow 0$ limit. On integration,

$$
\dot{S}_{4 \mathrm{~A}}=\frac{D_{0} k^{2}}{S(k)}\left[0.96 \phi^{2}\right] \text {. }
$$

The other nonzero part of $\dot{S}_{4}$ in the $k^{4}-0$ limit, here denoted $\dot{S}_{4 \mathrm{~B}}$, arises from the integral of Eq. (B10) with $c=e=0$ and $a=3$. One has

$$
\begin{aligned}
\nu_{330}(r, s)= & \frac{+\pi \sqrt{4} \pi}{64 r^{4} s^{4}}\left[5 r^{8}-4 r^{6} s^{2}-2 r^{4} s^{4}-4 r^{2} s^{6}+5 s^{8}+5 \sigma^{8}-20 s^{6} \sigma^{2}-12 r^{2} s^{4} \sigma^{2}-12 r^{4} s^{2} \sigma^{2}-20 r^{6} \sigma^{2}+30 s^{4} \sigma^{4}\right. \\
& \left.+36 r^{2} s^{2} \sigma^{4}+30 r^{4} \sigma^{4}-20 s^{2} \sigma^{6}-20 r^{2} \sigma^{6}\right] \text { for }|r+s| \geq \sigma ;|r-s| \leq \sigma,
\end{aligned}
$$

and

$$
\dot{S}_{4 \mathrm{~B}}=\frac{D_{0} k^{2}}{S(k)}\left[0.67 \phi^{2}\right]
$$

Arguments like those applied to Eq. (B12) demonstrate that for $c, e>0$ the integrals of Eq. (10) vanish as $k^{4}$ when $k \rightarrow 0$. 
${ }^{1}$ A. R. Altenberger and J. M. Deutch, J. Chem. Phys. 59, 894 (1973).

${ }^{2}$ G. D. J. Phillies, J. Chem. Phys. 60, 976 (1974).

${ }^{3}$ B. U. Felderhof, J. Phys. A: Math. Nucl. Gen. 11, 929 (1978).

${ }^{4}$ W. Hess and R. Klein, Physica A 85, 509 (1976),

${ }^{5}$ P. N. Pusey and R. J. A. Tough, in Dynamic Light Scattering and Velocimetry: Applications of Photon Correlation Spectroscopy, edited by R. Pecora (Plenum, New York, in fress).

${ }^{6}$ G. D. J. Phillies and P. R. Wills, J. Chem. Phys. 75, 508 (1981).

'(a) H. M. Fijnaut, C. Pathmamanoharan, E. A. Nieuwenhuis, and A. Vrij, Chem. Phys. Lett. 59, 351 (1978); (b) M. M.

Kops-Werkhoven and H. M. Fijnaut, in Light Scattering in Liquids and Macromolecular Solutions, edited by V. Degiorgio, M. Corti, and M. Giglio (Plenum, New York, 1980).

${ }^{8}$ (a) G. D. J. Phillies, J. Chem. Phys. 74, 260 (1981):

(b) Phys. Rev, A 24, 1939 (1981); (c) in American Association for Aerosol Research 1st Annual Meeting Symposium Volume, edited by $B$. Dahneke (in press).
${ }^{9}$ B. J. Ackerson, P. N. Pusey, and R. J. A. Tough, J. Chem. Phys. 76, 1279 (1982).

${ }^{10}$ B. U. Felderhof, Physica A 80, $172(1975)$; 84, 557, 569 (1976); 89,373 (1977).

${ }^{11}$ J. G. Kirkwood, R. L. Baldwin, P. J. Dunlop, L. J. Gosting, and G. Kegeles, J. Chem. Phys. 33, 1505 (1960).

${ }^{12} \mathrm{H}$. J. Silverstone and R. K. Moats, Phys. Rev. A 16, 1731 (1977).

${ }^{13}$ G. D. J. Phillies, J. Stat. Phys. (in press).

${ }^{14} \mathrm{G}$. K. Batchelor, J. Fluid Mech. 74, 1 (1976).

${ }^{15}$ B. J. Ackerson, J. Chem. Phys. 69, 684 (1978).

${ }^{16}$ W. Dieterich and I. Peschel, Physica A 95, 208 (1979).

${ }^{17} \mathrm{~J}$. A. Marqusee and J. M. Deutch, J. Chem. Phys. 73, 5397 (1980).

${ }^{18}$ G. D. J. Phillies, J. Chem. Phys. 74, 2436 (1981).

${ }^{19}$ D. A. McQuarrie, Statistical Mechanics (Harper and Row, New York, 1976).

${ }^{20}$ H. Faxen, Ann. Phys. (Leipzig) (IV) 8, 89 (1922); Doctoral thesis, University of Uppsala, 1921.

${ }^{21}$ A. R. Edmonds, Angular Momentum in Quantum Mechanics (Princeton University, Princeton, 1974). 